\title{
Selective use of postoperative neck radiotherapy in oral cavity and oropharynx cancer: a prospective clinical study
}

\author{
Miguel Martínez Carrillo ${ }^{1 *}$, Isabel Tovar Martín, Ildefonso Martínez Lara², \\ José Mariano Ruiz de Almodóvar Rivera ${ }^{3}$ and Rosario Del Moral Ávila'
}

\begin{abstract}
Background: In cervical postoperative radiotherapy, the target volume is usually the same as the extension of the previous dissection. We evaluated a protocol of selective irradiation according to the risk estimated for each dissected lymph node level.

Methods: Eighty patients with oral/oropharyngeal cancer were included in this prospective clinical study between 2005 and 2008. Patients underwent surgery of the primary tumor and cervical dissection, with identification of positive nodal levels, followed by selective postoperative radiotherapy. Three types of selective nodal clinical target volume (CTV) were defined: CTV0, CTV1, and CTV2, with a subclinical disease risk of $<10 \%, 10-25 \%$, and $25 \%$ and a prescribed radiation dose of $<35 \mathrm{~Gy}, 50 \mathrm{~Gy}$, and 66-70 Gy, respectively. The localization of node failure was categorized as field, marginal, or outside the irradiated field.

Results: A consistent pattern of cervical infiltration was observed in $97 \%$ of positive dissections. Lymph node failure occurred within a high-risk irradiated area (CTV1-CTV2) in 12 patients, marginal area (CTV1/CTVO) in 1 patient, and non-irradiated low-risk area (CTVO) in 2 patients. The volume of selective lymph node irradiation was below the standard radiation volume in 33 patients (mean of $118.6 \mathrm{cc}$ per patient). This decrease in irradiated volume was associated with greater treatment compliance and reduced secondary toxicity. The three-year actuarial nodal control rate was $80 \%$.

Conclusion: This selective postoperative neck irradiation protocol was associated with a similar failure pattern to that observed after standard neck irradiation and achieved a significant reduction in target volume and secondary toxicity.
\end{abstract}

Keywords: Oral cavity and oropharynx cancer, Postoperative radiotherapy, Selective neck irradiation, Lymph node

\section{Introduction}

Squamous cell cancer of the head and neck is diagnosed in 650,000 individuals annually worldwide and accounts for $5-10 \%$ of all malignant tumors [1]. The region with the highest incidence is Southern Europe, where there has been a gradual reduction in oral cancer and an increase in oropharyngeal cancer [2].

Squamous cell cancer of the head and neck follows a consistent and well-defined locoregional growth pattern.

\footnotetext{
* Correspondence: martinezcarrillo.miguel@gmail.com

'Department of Radiation Oncology, Virgen de las Nieves University Hospital, Avda. Fuerzas Armadas 4, Granada 18014, Spain

Full list of author information is available at the end of the article
}

The therapeutic decision depends on the lymph node metastatic status, because tumor control requires treatment of both the primary tumor and involved regional nodes [3].

Standard guidelines for selecting target volumes are based on knowledge of neck tumor distribution and dissemination patterns. The Memorial Sloan-Kettering Cancer Center described the pattern of cervical lymph node metastasis in 1077 naïve patients with head and neck cancer after performing 1118 neck dissections [4-6]. Neck dissection was performed in 343 of the 341 patients with a clinically NO neck cancer, i.e., bilateral dissection was only performed in two patients. Bilateral

\section{() BioMed Central}

(c) 2013 Martínez Carrillo et al.; licensee BioMed Central Ltd. This is an Open Access article distributed under the terms of the Creative Commons Attribution License (http://creativecommons.org/licenses/by/2.0), which permits unrestricted use, distribution, and reproduction in any medium, provided the original work is properly cited. 
neck dissection was carried out in 39 of the 736 patients with clinically positive nodes. Metastatic disease was confirmed in 33\% of elective neck dissections and $82 \%$ of therapeutic neck dissections. The distribution of pathologically confirmed metastatic lymph nodes varied according to the primary tumor site. In clinically N0 patients, metastatic lymph nodes were generally observed in levels I-III for oral cavity tumors (20\%, 17\% and 9\% respectively) and in levels II-IV for oropharyngeal tumors (25\%, 19\% and $8 \%$ respectively). A similar pattern was observed in the patients who underwent therapeutic neck dissection except for a higher density of adenopathies and significant pathologic infiltration of an additional level; among tumors of the oral cavity, $44 \%$ were in level I, 32\% in level II, $16 \%$ in level III, and $3 \%$ in levels IV; in oropharyngeal tumors, $15 \%$ were in level I, $71 \%$ in level II, $42 \%$ in level III, 27\% in level IV, and 9\% in level V. Pathological infiltration of level $\mathrm{V}$ was low, observing a single infiltration of level $\mathrm{V}$ in only one patient; it was below $1 \%$ when a single pathologically confirmed positive node was detected in levels I-III but increased to $16 \%$ when a single positive node was located in level IV. When more than one level was infiltrated, the likelihood of level $\mathrm{V}$ involvement progressively increased, reaching 40\% when levels I-IV were all involved. Pathological involvement of level I was observed in only $2 \%$ of clinical N0 patients with oropharyngeal tumors, increasing to $15 \%$ after therapeutic dissection. These observations illustrate the gradual and orderly infiltration of node levels in the neck.

Surgeons were the first to use the concept of selective treatment, performing selective dissections of the neck to remove only high-risk lymph node levels. This practice led surgeons to divide the neck into different levels according to anatomical references that can be easily identified during neck dissection procedures (i.e. major vessels, muscles, cartilages), known as the Robbins classification $[7,8]$. Selective neck dissection is currently the procedure of choice for elective neck surgery and for a specific group of neck-positive patients (selected N1 and N2 patients) [9] and is associated with a comparable nodal relapse rate to that observed in patients undergoing radical dissection [10-12].

The applicability of the Robbins classification to the definition of radiation target volumes remains controversial. The standardization of target volumes is challenging, because the relevant anatomical-surgical references are not always identifiable on CT or MR scans. References proposed in the 1990s for the radiological definition of neck lymph node levels were inconsistent [13-18], leading to the publication of consensus guidelines for N0 patients in 2003 [19]. In 2006, Gregoire et al. [20] proposed a modification of the definition of neck regions for postoperative radiation therapy, which otherwise appears to have received little research attention.
Target volume dose-dependent toxicity (e.g., oromucositis and dysphagia) can limit the correct uninterrupted administration of adjuvant radiation therapy [21]. Therapeutic compliance would be enhanced if target volumes and, therefore, secondary toxicity could be reduced while maintaining tumor control. Standardized neck dissection by levels can yield data that allow clinicians to adapt target volumes according to the estimated risk of involvement for each node level.

\section{Materials and methods}

For this study, we recruited all consecutive patients diagnosed with squamous cell cancer of the oral cavity or oropharynx between 2005 and 2008 who underwent surgical resection of the primary tumor, without gross residual disease, including unilateral or bilateral neck dissection with a report of histological results by neck level, and who received postoperative radiation therapy prescribed by a multidisciplinary team of head and neck specialists. Written informed consent was obtained from all recruited patients, and the trial was approved by the hospital ethics committee (Clinical Research Ethical Committe Virgen de las Nieves Hospital). Out of the 80 patients recruited, 5 were subsequently excluded after application of the following exclusion criteria: receipt of $<90 \%$ of the planned radiotherapy dose ( 1 patient), interruption of $>2$ weeks ( 1 patient), and the detection of cervical (2 patient), or local (1 patient) relapse during the course of the

\section{Table 1 Clinical and pathological features}

\begin{tabular}{|c|c|c|c|c|c|}
\hline Clinical & $\mathrm{n}$ & $\%$ & Pathological & $\mathrm{n}$ & $\%$ \\
\hline Gender & & & Infiltration & & \\
\hline Male & 56 & 75 & Vascular & 7 & 9 \\
\hline Female & 19 & 25 & Perineural & 13 & 17 \\
\hline Tumor location & & & Histological grade & & \\
\hline Oral cavity & 58 & 77 & G3 & 11 & 15 \\
\hline Oropharynx & 17 & 23 & G4 & 2 & 3 \\
\hline \multirow[t]{3}{*}{ Performance status ECOG 0-1 } & 70 & 93 & Resection margin & & \\
\hline & & & Close & 13 & 17 \\
\hline & & & Infiltrated & 9 & 12 \\
\hline Clinical stage & & & Pathological stage & & \\
\hline 1 & 4 & 5 & I & - & - \\
\hline$\|$ & 13 & 17 & $\|$ & - & - \\
\hline III & 21 & 28 & III & 24 & 32 \\
\hline IVa & 30 & 40 & IVa & 48 & 64 \\
\hline $\mathrm{IVb}$ & 7 & 9 & $\mathrm{IVb}$ & 3 & 4 \\
\hline \multirow[t]{2}{*}{ Clinical N+ } & 47 & 63 & Pathological N+ & 75 & 100 \\
\hline & & & Extracapsular spread & 31 & 41 \\
\hline
\end{tabular}

Median age of 62 years; range, 39-78 years.

ECOG = Eastern Cooperative Oncology Group; $\mathrm{N}+=$ Positive lymph node. 
Table 2 Nodal surgical procedure

\begin{tabular}{cccc}
\hline Cervical dissection (levels) & Ipsilateral & Contralateral & Total \\
\hline RND (I-V) & 7 & - & 7 \\
MRND (I-V) & 33 & 5 & 38 \\
SOHND (I-III) & 30 & 9 & 39 \\
LND (II-IV) & 5 & 1 & 6 \\
Total & 75 & 15 & 90 \\
\hline
\end{tabular}

Abbreviations: $\mathrm{RND}=$ radical neck dissection; $\mathrm{MRND}=$ modified radical neck dissection; SOHND = supraomohyoid neck dissection; LND = lateral neck dissection.

radiotherapy. The clinical-pathological characteristics of the patients in the final sample are described in Table 1.

Out of these 75 patients, 15 underwent both ipsilateral and contralateral node dissection. The type of dissection depended on the nodal stage, performing selective dissection in N0-N1 patients and dissection of all levels (I-V) in N2-N3 patients (Table 2).

Nodal levels were intraoperatively identified following standard guidelines and then stained and delineated using surgical clips. After en bloc resection, nodal levels were coded and sent for individual histopathological study with hematoxylin-eosin staining. Adenopathies $>3$ $\mathrm{mm}$ in diameter were identified, and a topographical diagram was created of the involved levels, the number and size of positive nodes, and the presence of extracapsular spread.

Following the standard protocol, postoperative radiotherapy was administered to the site of the primary tumor (close or infiltrated surgical margin, pT4, vascular/perineural invasion, histologically undifferentiated), the dissected side of the neck (pN1 with perinodal invasion, skip metastases or inadequate dissection, i.e., total nodal yield $\leq 5, \mathrm{pN} 2-\mathrm{N} 3$ ), and the clinical N0 contralateral non-dissected side of the neck with significant risk of subclinical involvement (considered for ipsilateral neck irradiation and/or primary in midline).

The clinical tumor volume (CTV) for elective neck radiotherapy without dissection (clinical contralateral N0) was delineated using an adaptation of consensus guidelines [19] with the above-mentioned modification by Gregoire et al. for postoperative neck radiotherapy [20]. This radiotherapy was administered to patients with at least two involved lymph nodes or with one involved lymph node generally associated with an unfavorable prognosis (at this level we only identified the infiltrated node, involved lymph nodes with a diameter $>3 \mathrm{~cm}$, or any extracapsular spread at any other dissected nodal level). N-pathological dissected levels adjacent to nodal levels with extracapsular spread and/ or $\mathrm{pN} 3$ were considered high-risk levels. When the involved lymph node was located at the boundary of two levels, both levels were considered high-risk. Levels situated between two or more high-risk levels were included in the target volume, regardless of their pathological status.

According to these criteria and the estimated risk for subclinical disease, we used three types of selective nodal CTV (Table 3) and two types of standard nodal CTV (all dissected neck levels) routinely used at our center for comparison of the results.

The Pinnacle 8.0 version 3, 3D planning system was used, and the treatment was administered by means of a Varian Clinac-D 2100C linear accelerator equipped with multileaf collimator. An isotropic margin of $5 \mathrm{~mm}$ was applied to the respective CTVs to generate the appropriate planning target volume (PTV). The dose variation in a PTV was not allowed to exceed $+7 \%$ or $-5 \%$ of the prescribed dose. Brain, brainstem, spinal cord, lens, retina, optic nerves, optic chiasm, parotid gland, and mandible, among other structures, were delineated on the CT image. The prescribed dose to the PTV was 50 Gy to the isocenter for PTV1 and a cumulative dose of 66-70 Gy for PTV2. Concomitant cisplatin-based chemotherapy was received by 36 patients with extracapsular spread and/or positive surgical margin $\left(100 \mathrm{mg} / \mathrm{m}^{2}\right.$ cisplatin every 3 weeks by 28 patients and $40 \mathrm{mg} / \mathrm{m}^{2}$ weekly by 8 ).

Post-treatment neck CT examinations with intravenous contrast were performed at 2, 6, 12, 18, 24 months and then annually, or when a relapse was suspected. Post-treatment nodal relapse was defined by the appearance of an adenopathy in the neck. A nodal relapse

Table 3 Selection of selective nodal clinical target volume (CTV)

\begin{tabular}{lccc}
\hline & Nodal CTV & Nodal CTV1 & Nodal CTV2 \\
\hline $\begin{array}{l}\text { Subclinical disease risk } \\
\text { Prescribed radiation dose }\end{array}$ & $<10 \%$ & $10 \%-25 \%$ & $>25 \%$ \\
Levels dissected & $<35$ Gy (a) & 50 Gy & $66-70$ Gy \\
& Negative level with sufficient dissection & Negative and/or positive level without risk factors \\
with insufficient dissection & $\begin{array}{c}\text { Positive level with } \\
\text { risk factors }\end{array}$ \\
Levels not dissected & Positive level without risk factors & Adjacent negative level to nodal CTV2 (ECS and/or pN3) & Ipsilateral: Non-dissected level adjacent to infiltrated \\
& - & dissected level & - \\
\end{tabular}



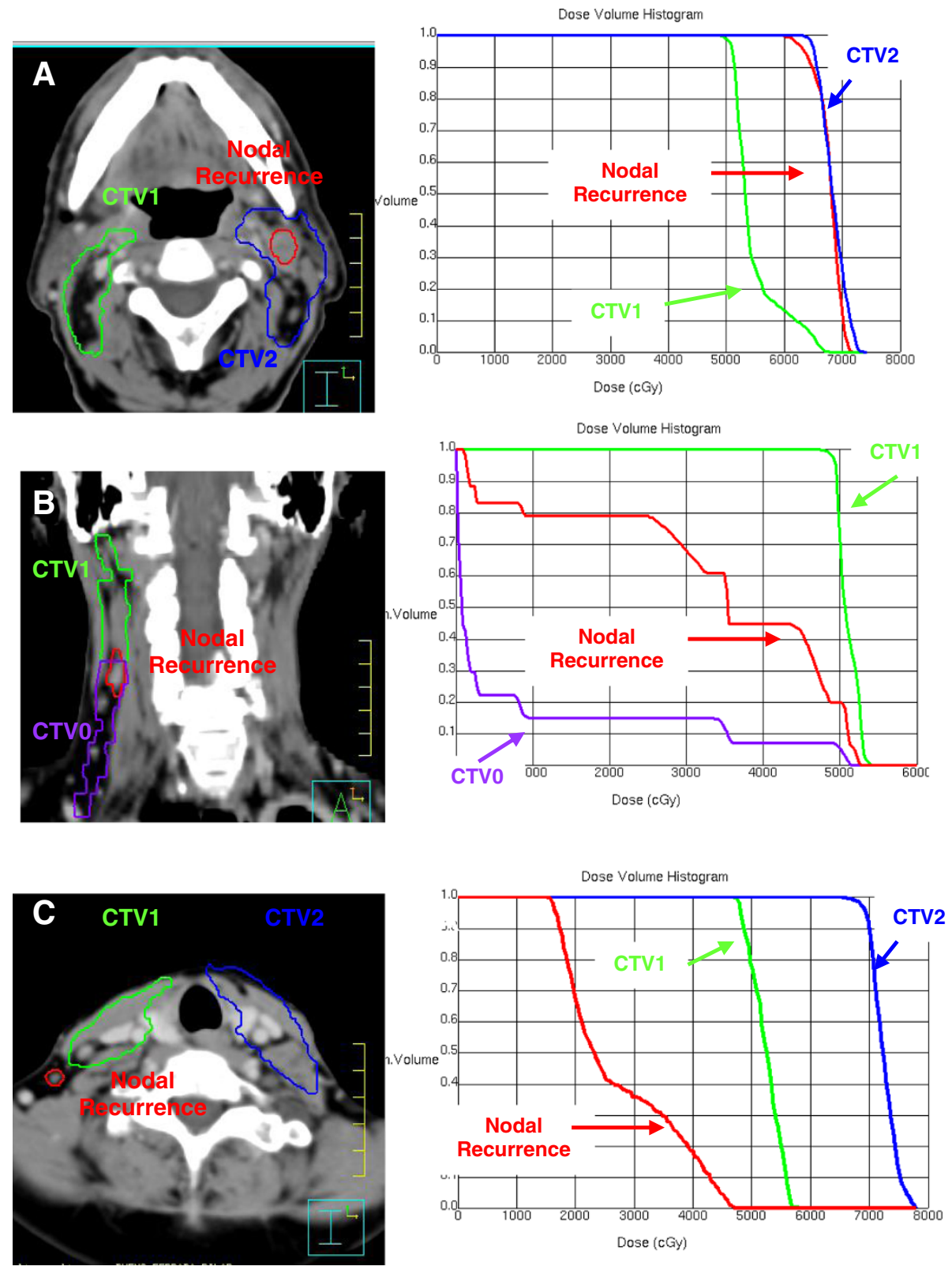

Figure 1 The radiologic imaging of nodal recurrence volume was co-registered with the pre-treatment $\mathrm{CT}$ data set used for treatment planning. (A) DVH analysis of nodal failure "in field". (B) DVH analysis of nodal failure "marginal field". (C) DVH analysis of nodal failure "out-field".

detected on the CT scan was confirmed by histological study, and a new CT-simulation scan was performed using the same thermoplastic mask and parameters (isocenter, thickness and number of radiological slices) to calculate the nodal relapse volume (NRV). After obtaining the dose-volume histograms, nodal relapse was classified as: within the irradiated field (>95\% of NRV within CTV1 or CTV2); within the margins of the irradiated field (20\%-95\% of NRV within CTV1 or CTV2); or outside the irradiated field $(<20 \%$ of NRV within CTV1 or CTV2) (Figure 1).
Actuarial locoregional control and survival rates were calculated by using the Kaplan-Meier method. SPSS version 19 (IBM, Chicago IL) was used for all data analyses.

\section{Results}

As depicted in Figure 2, 1,545 lymph nodes were dissected: 1,294 nodes in ipsilateral dissections and 251 in contralateral dissections, distributed over the five neck levels. Metastasis to at least one of the examined lymph nodes was observed in all 75 ipsilateral necks 


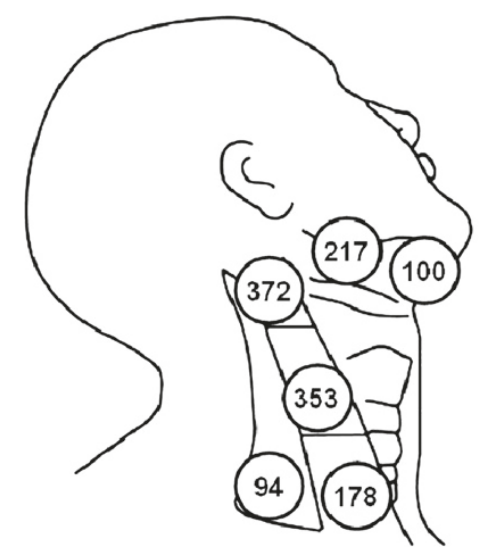

Ipsilateral

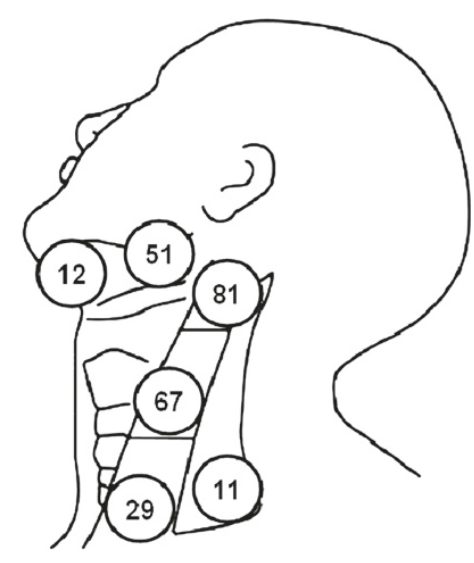

Contralateral

Figure 2 Quantification of nodal levels dissected.

dissected, which evidenced a total of 121 involved (positive) levels, i.e., a mean of 1.6 positive ipsilateral node levels per patient (1-4). These 121 levels contained a total of 183 metastatic adenopathies, i.e., a mean of 1.5 positive nodes per involved level (1-7). Level II was the most frequently involved in the oral cavity (16/31) and oropharynx (37/90) tumors; among the oral cavity cancers, level Ib was most frequent in tumors of the floor of the mouth (14/27). A regular neck dissemination pattern to levels I to III was observed in 73 (97\%) of the 75 ipsilateral dissections, starting in levels Ia/Ib in 39 dissections, level II in 30, and level III in 4. In the other two ipsilateral dissections, one showed involvement of levels Ib, II and IV but not of level III and the other involvement of levels II and V but not of levels III or IV.

Only one case of metastasis to a contralateral node was detected (to levels II and Ib).

Post-treatment nodal failure was observed in 15 of the 75 patients, local failure in 9, distal failure in 7 , and a second primary tumor in 3 (Table 4). All nodal relapse cases were located in the ipsilateral dissected neck. Nodal failure was within high-risk irradiated areas (nodal CTV1, CTV2) in 12 patients, in a marginal area (nodal CTV1/CTV0) in 1 patient, and outside the irradiation area (nodal CTV0) in the remaining 2 patients, who therefore did not receive post-treatment radiation therapy in this zone (Table 5).

The dosimetric study of nodal failures revealed a mean NRV of 4.4 cc $(1.9-10.2 \mathrm{cc})$. Mean, minimal, and maximal doses were, respectively, $6518 \mathrm{cGy}, 6420 \mathrm{cGy}$, and 6894 cGy for patients with nodal failure in the irradiated field; 3091 cGy, 590 cGy, and 5101 cGy for those with failure within the margins of the field; and 2357 cGy, 320 cGy, and 4421 cGy for patients with nodal failure outside the radiation field (Table 5).

Out of the 75 patients in the study, 33 (44\%) received postoperative selective nodal irradiation, with a mean reduction in target volume of 118.6 cc per patient (21.9 cc-234.7 cc), i.e., nodal CTV0, with a mean reduction of 1.8 levels per patient. Dose stratification by levels allowed us to administer dose-escalated radiotherapy from a dose of $50 \mathrm{~Gy}$, only applying 66-70 Gy in highrisk levels. Out of the total of 186 irradiated levels, $40 \%$ received a high dose (66-70 Gy: nodal CTV2) and 60\% a low dose (50 Gy: nodal CTV1).

After a mean follow-up of 32 months, 45 patients (60\%) were alive and disease-free, 2 were alive with disease (3\%), 22 patients died from the tumor (29\%), and

Table 4 Pattern of treatment failure

\begin{tabular}{lcccc}
\hline Failure & $\mathbf{n}(\%)$ & Post-treatment months (range) & Prognostic factors & Localization $(\mathbf{n})$ \\
\hline Nodal failure & $15(20)$ & $10.2(6-19)$ & Age $>62$ yrs $(\mathrm{p} \mathrm{0.002)}$ \\
Local failure & $9(12)$ & $11.4(7-23)$ & Oral c. (tongue) $(\mathrm{p}=0.042)$ & - \\
Distal failure & $7(9)$ & $11.7(6-20)$ & - & Lung (5) \\
& & & Bone (1) \\
& & & Skin (1) \\
Second primary tumor & $3(4)$ & $25(11-46)$ & Lung (2) \\
\end{tabular}


Table 5 Failure pattern and dosimetric characteristics in patients with nodal recurrence

\begin{tabular}{|c|c|c|c|c|c|c|c|}
\hline Patient $n^{\circ}$ & $\begin{array}{l}\text { Localization } \\
\text { nodal failure }\end{array}$ & XRT volume & $\begin{array}{c}\text { Failure } \\
\text { (month) }\end{array}$ & $\begin{array}{l}\text { Volume } \\
\text { (cc) }\end{array}$ & $\begin{array}{l}\text { Minimum dose } \\
\text { (cGy) }\end{array}$ & $\begin{array}{l}\text { Maximum dose } \\
\text { (cGy) }\end{array}$ & $\begin{array}{c}\text { Mean dose } \\
\text { (cGy) }\end{array}$ \\
\hline 1 & II-III ipsilateral & Nodal CTV2 & 6 & 9.1 & 6498.2 & 6980.0 & 6602.9 \\
\hline 2 & III-IV ipsilateral & Nodal CTV2 & 12 & 3.45 & 6489.9 & 6995.9 & 6629.5 \\
\hline \multirow[t]{2}{*}{3} & II ipsilateral & Nodal CTV1 & 14 & 2.8 & 4399.0 & 6551.2 & 5554.1 \\
\hline & & (Nodal CTV2 marginal) & & & & & \\
\hline 4 & III ipsilateral & Nodal CTV0 & 9 & 9.35 & 175.1 & 3113.2 & 1191.4 \\
\hline \multirow[t]{2}{*}{5} & III ipsilateral & Nodal CTV1 & 8 & 1.83 & 5685.5 & 7005.2 & 6535.1 \\
\hline & II ipsilateral & Nodal CTV2 & 8 & 2.51 & 6652.8 & 6741.6 & 6701.8 \\
\hline 6 & II ipsilateral & Nodal CTV2 & 7 & 3.5 & 7009.1 & 7255.1 & 7149.8 \\
\hline 7 & V ipsilateral & Nodal CTV0 & 19 & 3.1 & 195.0 & 5050.1 & 2789.8 \\
\hline 8 & |b-|l ipsilateral & Nodal CTV2 & 8 & 3.35 & 6561.1 & 7171.2 & 6805.5 \\
\hline \multirow[t]{2}{*}{9} & II-III ipsilateral & Nodal CTV0 & 17 & 2.9 & 589.9 & 5101.0 & 3091.0 \\
\hline & & (nodal CTV1 marginal) & & & & & \\
\hline \multirow[t]{2}{*}{10} & Ib ipsilateral & Nodal CTV1 & 11 & 2.8 & 6491.0 & 7001.0 & 6682.2 \\
\hline & II ipsilateral & Nodal CTV2 & 11 & 1.9 & 4901.0 & 6281.2 & 5402.3 \\
\hline 11 & II ipsilateral & Nodal CTV2 & 7 & 9.8 & 6595.3 & 6798.4 & 6639.0 \\
\hline 12 & Ib ipsilateral & Nodal CTV2 & 7 & 2.9 & 6598.2 & 6792.0 & 6689.3 \\
\hline 13 & Il ipsilateral & Nodal CTV2 & 8 & 2.1 & 6601.4 & 6850.1 & 6602.2 \\
\hline 14 & Ib ipsilateral & Nodal CTV2 & 11 & 2.8 & 6599.0 & 6899.4 & 6598.0 \\
\hline 15 & Ib ipsilateral & Nodal CTV2 & 10 & 10.2 & 6278.7 & 7195.5 & 6658.9 \\
\hline
\end{tabular}

Abbreviations: CTV0 = low-risk clinical target volume; CTV1 = moderate-risk clinical target volume; CTV2 $=$ high-risk clinical target volume.

6 died from other causes (8\%). Nodal relapse-free survival rates were $84 \%$ at 12 months and $79 \%$ at 36 months.

\section{Discussion}

Following positive nodal dissection, standard target volume radiotherapy includes all node levels considered to be high-risk by the surgeon, coinciding with the extent of the dissection and including retropharyngeal lymph nodes, which are not usually dissected [13,22]. Selective nodal irradiation hypothetically offers a decrease in target volume with no increase in marginal failures, considering the neck as a set of volumes for mapping high-risk neck regions. Evidently, selective nodal irradiation would not significantly reduce the target volume in patients with bulky metastasis and/or multiple neck level involvement.

It proved possible to administer postoperative selective nodal irradiation to nearly half of the present series of 75 head-and-neck cancer patients, obtaining a significant reduction in target volume. Dose-volume histogram analysis showed that the dose for recurrent nodal disease was comparable to or higher than the target prescription. Nodal failure was predominantly observed in the high-dose volume (CTV2), suggesting the need to discern a radiation-resistant subpopulation within CTV2.

Given the likely relationship of radiotherapy-induced toxicity with the target volume and administered dose, this toxicity might be significantly reduced by a selective irradiation approach. In the TROG 91:01 trial, which included 350 head and neck cancer patients, Poulsen et al. [23] reported that the target volume in the second phase of treatment had the strongest influence on the development of odynophagia and need for aggressive nutritional intervention (gastrotomy or insertion of nasogastric tube), finding that the likelihood of developing grade-4 (G-4) odynophagia was $36 \%$ when the volume was $>82 \mathrm{~mm}$ and $16 \%$ when it was $<18 \mathrm{~mm}(\mathrm{p}=0.0001)$.

In our series, acute toxicity most frequently presented as mucositis and dysphagia in the patients undergoing radiotherapy (G-3 mucositis in 16\%; G-3 dysphagia in $14 \%)$ and in those treated with concomitant chemoradiotherapy (G-3 mucositis in 27\%; G-4 mucositis in 6\%; G-3 dysphagia in 21\%). Out of the 75 patients, only $1.3 \%$ received $<95 \%$ of the prescribed radiation dose; the dose administered to neck volumes represented $98.4 \%$ of the prescribed dose (nodal CTV1: 99.6\%, nodal CTV2: 98\%), $90.6 \%$ completed their radiation therapy plan with no more than one day of interruption, and all completed 
the plan with less than six days of interruption; only $3.5 \%$ of interruptions were secondary to toxicity, a lower percentage than in previous reports [24,25]. In the study based on SEER's (Surveillance, Epidemiology and End Results) [26] database, which included 5,086 patients with head-and-neck cancer, $29.6 \%$ of the patients treated with surgery and radiotherapy showed treatment discontinuity and/or non-adherence (33.3\% of those with oral cancer and $33.5 \%$ of those with oropharyngeal cancer).

The development and incorporation of postoperative selective nodal irradiation techniques into clinical practice requires the routine performance of neck dissection by level. The possibility that the results may be inferior to those obtained with en bloc dissection was refuted by Upile et al., who found no difference in total yield between specimens sent to the histopathologist en bloc or divided into levels [27].

The variability in the number of nodes obtained from neck dissection can only be partially attributed to anatomical differences among patients, given that previous studies indicated a minimum of 28 nodes on each side of the dissected neck [28]. The primary cause of nodal variability is generally related to the surgical technique. Although the boundaries of radical dissection are defined in consensus guidelines $[7,8]$, they may be modified by surgeons based on their own professional experience or because the terminology used to define the different types and dimensions of neck dissection is inconsistent. A substantial reduction in nodal variability can be expected if surgeons strictly adhere to the anatomical boundaries established in consensus guidelines for dissection procedures. The number of nodes is usually lower in selective procedures, such as supraomohyoid or lateral dissection, due to the smaller number of dissected levels [9-12]. The amount of lymphoid tissue dissected for histological study by level should always be the same, regardless of the type of dissection (radical, modified, or selective). In the present series, there was a minimal variability in the mean number of nodes identified in each level as a function of the type of dissection.

The histopathological report plays a key role in the assessment of the dissected specimen. The standard procedure for studying a dissected node after hematoxylineosin staining is to remove a longitudinal section for examination under light microscopy to detect metastatic deposits [29]. These partial findings are then extrapolated to the remaining nodal volume. Although an incontrovertible diagnosis would be provided by examination of $5-\mu \mathrm{m}$ sections of the whole node, as in sentinel-node techniques, this is a laborious and costly procedure, and the clinical relevance of the detection of occult subpathological metastatic deposits remains unclear [29].

Radiotherapy oncologists have devoted considerable research efforts to measures for the protection of normal tissues from radiation, with a focus on minimizing their exposure. However, there have been few attempts to determine the target volume for irradiation in each specific situation, and no standardized patterns of lymph node relapse after postoperative neck radiation therapy have been established, which may be because target volumes generally include all lymph nodes on both sides of the neck. To our best knowledge, the present investigation offers one of the few descriptions of a selective nodal irradiation protocol for patients undergoing surgery for oral cavity and pharyngeal cancer according to the estimated risk for each nodal level.

\section{Conclusion}

In conclusion, nodal involvement in oral cavity and pharyngeal cancer follows a predictable and gradual pattern across the different neck levels, supporting the selective neck irradiation approach. The availability of a reliable postoperative nodal-involvement pattern by level allows clinicians to determine target volumes according to the estimated risk for each nodal level. The neck failure pattern obtained after a postoperative selective nodal irradiation protocol does not differ from that obtained after a standard irradiation protocol. Nodal recurrence is most frequently detected in high-risk nodal levels receiving high irradiation doses. The target volume with selective nodal radiotherapy is significantly lower than the target volume with a standard nodal radiotherapy protocol.

Selective nodal irradiation of the neck is not yet a standard treatment. However, greater understanding of the natural history of the tumors and the availability of increasingly sophisticated radiotherapy techniques will facilitate further trials to support oncologists in the optimal selection of high-risk target volumes.

\section{Competing interests \\ The authors declare that they have no competing interest.}

\section{Authors' contributions}

MMC conceived and developed the project and supervised RT applications. IML participated in this design and coordination. MMC, ITM, IML and RDA collected the data. JMRAR critically reviewed the manuscript. All authors read and approved the final manuscript.

\section{Acknowledgements}

This work was supported, in part, by Grants-in-Aid for Scientific Research from the Health Andalusian Authority PI-SAS-209/04.

\section{Author details}

${ }^{1}$ Department of Radiation Oncology, Virgen de las Nieves University Hospital, Avda. Fuerzas Armadas 4, Granada 18014, Spain. ²Department of MaxilloFacial Surgery, Virgen de las Nieves University Hospital, Granada, Spain. ${ }^{3}$ Department of Radiology and Physical Medicine, University of Granada, Granada, Spain.

Received: 21 October 2012 Accepted: 16 February 2013 Published: 28 April 2013

\section{References}

1. GLOBOCAN 2008. 2008. http://www-dep.iarc.fr/. 
2. de Souza DL, de Camargo CM, Pérez MM, Curado MP: Trends in the incidence of oral cavity and oropharyngeal cancers in Spain. Head Neck 2012, 34:649-654.

3. Ferlito A, Rinaldo A, Robbins KT, Silver CE: Neck dissection: Past, present and future? J Laryngol Otol 2006, 120:87-92.

4. Candela FC, Kothari K, Shah JP: Patterns of cervical node metastases from squamous carcinoma of the oropharynx and hypopharynx. Head Neck 1990, 12:197-203.

5. Shah JP: Patterns of cervical lymph node metastasis from squamous carcinomas of the upper aerodigestive tract. Am J Surg 1990, 160:405-409.

6. Shah JP, Candela FC, Poddar AK: The patterns of cervical lymph node metastases from squamous carcinoma of the oral cavity. Cancer 1990, 66:109-113.

7. Robbins KT, Medina JE, Wolfe GT, Levine PA, Sessions RB, Pruet CW: Standardizing neck dissection terminology. Official report of the Academy's committee for head and neck surgery and oncology. Arch Otolaryngol Head Neck Surg 1991, 117:601-605.

8. Robbins KT, Clayman G, Levine PA, Medina J, Sessions R, Shaha A, et al: Neck dissection classification update. Revisions proposed by American Head and Neck Society and the American Academy of Otolaryngology Head and Neck Surgery. Arch Otolaryngol Head Neck Surg 2002, 128:751-758.

9. Chepeha DB, Hoff PT, Taylor RJ, Bradford CR, Teknos TN, Esclamado RM: Selective neck dissection for the treatment of neck metastasis from squamous cell carcinoma of the head and neck. Laryngoscope 2002, 112:434-438.

10. Ferlito A, Rinaldo A, Silver CE, Gourin CG, Shah JP, Clayman GL, et al: Elective and therapeutic selective neck dissection. Oral Oncol 2006, 42:14-25.

11. Ferlito A, Buckley JG, Shaha AR, Rinaldo A: Rationale for selective neck dissection in tumors of the upper aerodigestive tract. Acta Otolaryngol 2001, 121:548-555.

12. Buckley JG, Feber T: Surgical treatment of cervical node metastases from squamous carcinoma of the upper aerodigestive tract: Evaluation of the evidence for modifications of neck dissections. Head Neck 2001, 23:907-915.

13. Grégoire V, Coche E, Cosnard G, Hamoir M, Reychler H: Selection and delineation of lymph node target volume in head and neck conformal radiotherapy. Proposal for standardizing terminology and procedure based on the surgical experience. Radiother Oncol 2000, 56:135-150.

14. Som PM, Curtin HD, Mancuso AA: An imaging based classification for the cervical nodes designed as an adjunct to recent clinically based nodal classifications. Arch Otolaryngol Head Neck Surg 1999, 125:388-396.

15. Nowak PJ, Wijers OB, Lagerwaard FJ, Levendag PC: A three-dimensional CT-based target definition for elective irradiation of the neck. Int J Radiat Oncol Biol Phys 1999, 45:33-39.

16. Gregoire V, Braaksma M, Muller K, Gregoire V, Braaksma M, Muller K: CT-based delineation of the neck node CTV in head and neck conformal radiotherapy (3D-CRT/IMRT): The Bussels-Rotterdam consensus guidelines. Radiother Oncol 2002, 64(Suppl.1):S251-S252.

17. Palazzi M, Soatti C, Bianchi E, Alterio D, Baio A, Bocci C, et al: Guidelines for the delineation of nodal regions of the head and neck on axial tomography images. Tumori 2002, 88:355-360.

18. Van Triest B, Kaanders J, Jooster F: Optimization of the definition of cervical lymph node regions for 3D radiotherapy in head and neck cancer. Radiother Oncol 2002, 64(Suppl 1):S90.

19. Grégoire V, Levendag P, Ang KK, Bernier J, Braaksma M, Budach V, et al: $\mathrm{CT}$-based delineation of lymph node levels and related CTVs in the node-negative neck: DAHANCA, EORTC, GORTEC, NCIC, RTOG consensus guidelines. Radiother Oncol 2003, 69:227-236.

20. Grégoire V, Eisbruch A, Hamoir M, Levendag P: Proposal for the delineation of the nodal CTV in the node positive and the postoperative neck. Radiother Oncol 2006, 79:15-20.

21. Russo G, Haddad R, Posner M, Machtay M: Radiation treatment breaks and ulcerative mucositis in head neck cancer. Oncologist 2008, 13:886-898.

22. Chao KS, Ozyigit G, Tran BN, Cengiz M, Dempsey JF, Low DA: Patterns of failure in patients receiving definitive and postoperative IMRT for head and neck cancer. Int J Radiat Oncol Biol Phys 2003, 55:312-321.

23. Poulsen MG, Riddle B, Keller J, Porceddu SV, Tripcony L: Predictors of acute grade 4 swallowing toxicity in patients with stages III and IV squamous carcinoma of the head and neck treated with radiotherapy alone. Radiother Oncol 2008, 87:253-259.
24. Maciá I, Garau M, Solé Monné J, Cambra Serés MJ, Monfà Binefa C, Peraire LM: Compliance to the prescribed overall treatment time (OTT) of curative radiotherapy in normal clinical practice and impact on treatment duration of counteracting short interruptions by treating patients on Saturdays. Clin Transl Oncol 2009, 11:302-311.

25. James ND, Williams MV, Summers ET, Jones K, Cottier B: The management of interruptions to radiotherapy in head and neck cancer: an audit of the effectiveness of National Guidelines. Clin Oncol 2008, 20:599-605.

26. Fesinmeyer MD, Mehta V, Tock L, Blough D, McDermott C, Ramsey SD: Completion of radiotherapy for local and regional head and neck cancer in Medicare. Arch Otolaryngol Head Neck Surg 2009, 135:860-867.

27. Upile T, Jerjes $W$, Nouraei SA, Singh S, Clarke P, Rhys-Evans P, et al: How we do it: a method of neck dissection for histopathological analysis. BMC Surg 2007, 7:21

28. Friedman M, Lim JW, Dickey W, Tanyeri H, Kirshenbaum GL, Phadke DM, et al: Quantification of lymph nodes in selective neck dissection. Laryngoscope 1999, 109:268-370.

29. Debaney K, Rinaldo A, Ferlito A: Micrometastases in cervical lymph nodes from patients with squamous carcinoma of the head and neck: should they be actively sought? Maybe. Am J Otolaryngol 2007, 28:271-274.

\section{doi:10.1186/1748-717X-8-103}

Cite this article as: Martínez Carrillo et al:: Selective use of postoperative neck radiotherapy in oral cavity and oropharynx cancer: a prospective clinical study. Radiation Oncology 2013 8:103.

\section{Submit your next manuscript to BioMed Central and take full advantage of:}

- Convenient online submission

- Thorough peer review

- No space constraints or color figure charges

- Immediate publication on acceptance

- Inclusion in PubMed, CAS, Scopus and Google Scholar

- Research which is freely available for redistribution 